\title{
Incoherent $\rho$ meson photoproduction in ultraperipheral nuclear collisions at the CERN Large Hadron Collider
}

\author{
V. Guzey $\odot$, E. Kryshen $\odot$, and M. Zhalov $\odot$ \\ National Research Center “Kurchatov Institute”, Petersburg Nuclear Physics Institute (PNPI), Gatchina, 188300, Russia
}

(Received 6 March 2020; accepted 13 July 2020; published 30 July 2020)

\begin{abstract}
Using the Gribov-Glauber model for photon-nucleus scattering and a generalization of the vector meson dominance model for the hadronic structure of the photon, we make predictions for the cross section of incoherent $\rho$ photoproduction in $\mathrm{Pb}-\mathrm{Pb}$ ultraperipheral collisions in the Large Hadron Collider kinematics. We find that the effect of the inelastic nuclear shadowing is significant and leads to an additional $25 \%$ suppression of the incoherent cross section. Comparing our predictions to those of the STARlight Monte Carlo framework, we observe very significant differences.
\end{abstract}

DOI: 10.1103/PhysRevC.102.015208

\section{INTRODUCTION}

Ultraperipheral collisions (UPCs) of heavy ions at the Relativistic Heavy Ion Collider (RHIC) and the Large Hadron Collider (LHC) give an opportunity to explore high-energy nuclear physics with beams of quasireal photons [1]. Indeed, UPCs of relativistic ions are characterized by large transverse distances $b$ (impact parameters) between the centers of colliding nuclei of radii $R_{A}$ and $R_{B}, b \gg R_{A}+R_{B}$, so that the strong nucleus-nucleus interaction is suppressed leading to dominance of the photon-nucleus interactions involving quasireal photons emitted by the colliding nuclei. These photons have a wide energy spectrum extending into a $\mathrm{TeV}$ range for the LHC beam energies (in the target nucleus rest frame) and the high flux scaling as $Z^{2}$ ( $Z e$ is the electric charge of the photon-emitting nucleus). Among many exciting directions of UPC studies, coherent and incoherent photoproduction of light vector mesons on nuclei allow one to investigate soft meson-nucleus interactions at high energies in the kinematic domain unavailable with fixed nuclear targets. While it is generally understood and accepted that the strong suppression of cross sections of high-energy soft hadron-nucleus scattering is due to the nuclear shadowing effect arising as the result of multiple interactions of the projectile with target nucleons [2-4], the practical implementation of this mechanism in coherent and incoherent photoproduction of light vector mesons in UPCs differs in the literature [5-16]. In particular, the open questions include the generalization of the naive vector meson dominance (VMD) model to the case of photon-nucleus interactions, the comparatively large

Published by the American Physical Society under the terms of the Creative Commons Attribution 4.0 International license. Further distribution of this work must maintain attribution to the author(s) and the published article's title, journal citation, and DOI. Funded by $S C O A P^{3}$. uncertainty in the experimentally determined magnitude of the vector meson-nucleon cross section, and the size and energy dependence of the inelastic (Gribov) shadowing correction. Also, attempts to calculate the nuclear cross sections with a precision better than $10 \%$ immediately raise the issues of the dependence of the nuclear cross section on the choice of the nuclear wave function, the role of short-range nucleonnucleon correlations, and the finite range (radius) of hadronnucleon interactions.

This paper extends our earlier work [8] on coherent $\rho$ meson photoproduction on nuclei in UPCs of heavy ions in the kinematics of the LHC to the case of incoherent $\rho$ photoproduction (the target nucleus breaks up), where differences among scarce theoretical predictions seem to be very significant. Also, such an analysis is topical in view of anticipated LHC experimental data on this process. In particular, we calculate the incoherent $\mathrm{PbPb} \rightarrow \rho P b A^{\prime}$ UPC cross section ( $A^{\prime}$ stands for the products of dissociation of the nuclear target) as a function of the rapidity $y$ at the invariant center-of-mass energy $\sqrt{s_{N N}}=5.02 \mathrm{TeV}$ and as a function of $\sqrt{s_{N N}}$ at the central rapidity $y=0$. We also present our results for the incoherent photoproduction cross section $\gamma P b \rightarrow \rho A^{\prime}$ as a function of the photon-nucleon energy $W_{\gamma p}$.

Our results show that within the adopted model for the hadronic structure of the incident photon, the effect of the inelastic nuclear shadowing in the incoherent cross sections is significant. This observation complements a similar conclusion in the coherent case [8], which was confirmed by a comparison of our calculations with the ALICE measurements $[17,18]$. We also find very significant differences of our results for the incoherent photoproduction cross section from predictions of the STARlight Monte Carlo framework, which is frequently used in the UPC experimental data processing and analysis [14].

The remainder of the paper is structured as follows. In Sec. II, we present expressions for the UPC, incoherent, and coherent nuclear photoproduction cross sections, which we used in our analysis. Our predictions for the resulting 
cross sections in the LHC kinematics, their discussion, and comparison to the STARlight model are given in Sec. III. Finally, we summarize and draw conclusions in Sec. IV.

\section{INCOHERENT VECTOR MESON PHOTOPRODUCTION IN HEAVY-ION ULTRAPERIPHERAL COLLISIONS}

In the case of incoherent photoproduction of $\rho$ mesons in symmetric UPCs of ions $A$ and using the equivalent photon approximation [19], the UPC cross section can be written in the following form [1]:

$\frac{d \sigma_{A A \rightarrow \rho A A^{\prime}}}{d y}=N_{\gamma / A}(y) \sigma_{\gamma A \rightarrow \rho A^{\prime}}(y)+N_{\gamma / A}(-y) \sigma_{\gamma A \rightarrow \rho A^{\prime}}(-y)$

where $N_{\gamma / A}(y)$ is the photon flux; $y$ is the rapidity of the produced $\rho$ meson. Because each ion can serve as a source of photons and a target, Eq. (1) contains two contributions corresponding to the right-moving photon source (first term) and the left-moving source (second term), respectively. Equation (1) implies the situation (experimental setup), when the final state contains only a (reconstructed) $\rho$ meson, two large rapidity gaps, and no special requirement is imposed on the number of forward nucleons, which are emitted in the nuclear breakup. However, requiring that UPCs are accompanied by forward neutron emission detected by zero-degree calorimeters (ZDCs) allows one to disentangle with a high probability and a reasonable accuracy the two terms in Eq. (1); see the discussion in Ref. [20].

The photon flux $N_{\gamma / A}(y)$ in Eq. (1) is given by the convolution of the photon flux produced by a fast moving ion at the distance $\vec{b}$ from its center, $N_{\gamma / A}(\omega, \vec{b})$ [21], with the probability not to have strong interactions at given $\vec{b}, \Gamma_{A A}(b)$,

$$
N_{\gamma / A}(y)=\int d^{2} \vec{b} N_{\gamma / A}(\omega, \vec{b}) \Gamma_{A A}(\vec{b})
$$

where

$$
\Gamma_{A A}(\vec{b})=\exp \left(-\sigma_{N N} \int d^{2} \vec{b}_{1} T_{A}\left(\vec{b}_{1}\right) T_{A}\left(\vec{b}-\vec{b}_{1}\right)\right) .
$$

In Eq. (3), $T_{A}(\vec{b})=A \int_{-\infty}^{\infty} d z \rho_{A}(\vec{b}, z)$ is the nuclear optical density, where $\rho_{A}$ is the nuclear density, which we calculated using the Hartree-Fock-Skyrme approach [22]; $\sigma_{N N}$ is the energy-dependent nucleon-nucleon total cross section [23].

Combining the vector meson dominance (VMD) model for the $\gamma N \rightarrow \rho N$ amplitude with the high-energy optical limit of the Glauber model and using the completeness (closure) of the nuclear final states $A^{\prime}$, the expression for the cross section of incoherent photoproduction of $\rho$ mesons (and other vector mesons amenable to the VMD model) can be presented in the following form [24]:

$$
\sigma_{\gamma A \rightarrow \rho A^{\prime}}^{\text {Glauber }}=\left(\frac{e}{f_{\rho}}\right)^{2} \int d^{2} \vec{b}\left[\left\langle 0\left|\Gamma_{A}^{\dagger}(b) \Gamma_{A}(b)\right| 0\right\rangle-\left\langle 0\left|\Gamma_{A}^{\dagger}(b)\right| 0\right\rangle\left\langle 0\left|\Gamma_{A}(b)\right| 0\right\rangle\right],
$$

where $f_{\rho}$ is the photon-meson coupling fixed by the $\rho \rightarrow e^{+} e^{-}$decay width, $f_{\rho}^{2} /(4 \pi)=2.01 \pm 0.1$; the notation $\langle|\ldots|\rangle$ stands for the integration with the ground-state nuclear wave function squared (nuclear density). In Eq. (4), $\Gamma_{A}(b)$ is the $\rho$-nucleus scattering amplitude in the impact parameter space (profile function),

$$
\Gamma_{A}(b)=1-\prod_{i=1}^{A}\left(1-\Gamma_{N}\left(b-s_{i}\right)\right)
$$

which is expressed through the $\rho$-nucleon amplitudes $\Gamma_{N}$,

$$
\Gamma_{N}\left(b-s_{i}\right)=\frac{\sigma_{\rho N}}{4 \pi B} e^{-\left(b-s_{i}\right)^{2} /(2 B)},
$$

where $s_{i}$ is the transverse coordinate of the $i$ th nucleon; $\sigma_{\rho N}$ is the total $\rho$ meson-nucleon cross section; $B$ is the slope of the $t$ dependence of the $\rho N \rightarrow \rho N$ cross section. Note that in the high-energy limit, one can safely neglect the longitudinal momentum transfer to nucleons and the nucleon ordering. Substituting Eqs. (5) and (6) in Eq. (4) and assuming independent nucleons in the nuclear wave function, one obtains

$$
\begin{aligned}
& \left\langle 0\left|\Gamma_{A}^{\dagger}(b) \Gamma_{A}(b)\right| 0\right\rangle-\left\langle 0\left|\Gamma_{A}^{\dagger}(b)\right| 0\right\rangle\left\langle 0\left|\Gamma_{A}(b)\right| 0\right\rangle=\left(1-\frac{\sigma_{\rho N}}{A} T_{A}(b)+\frac{\sigma_{\rho N}^{2}}{16 \pi B A} T_{A}(b)\right)^{A}-\left(1-\frac{\sigma_{\rho N}}{2 A} T_{A}(b)\right)^{2 A} \\
& \quad=\exp \left[-\sigma_{\rho N} T_{A}(b)+\frac{\sigma_{\rho N}^{2}}{16 \pi B} T_{A}(b)\right]-\exp \left[-\sigma_{\rho N} T_{A}(b)\right]=\left(1-\exp \left[-\frac{\sigma_{\rho N}^{2}}{16 \pi B} T_{A}(b)\right]\right) \exp \left[-\sigma_{\rho N} T_{A}(b)+\frac{\sigma_{\rho N}^{2}}{16 \pi B} T_{A}(b)\right] \\
& \approx \frac{\sigma_{\rho N}^{2}}{16 \pi B} T_{A}(b) \exp \left[-\left(\sigma_{\rho N}-\frac{\sigma_{\rho N}^{2}}{16 \pi B}\right) T_{A}(b)\right] .
\end{aligned}
$$

In the last line, we expanded in powers of the elastic $\rho$-nucleon cross section $\sigma_{\rho N}^{\mathrm{el}}=\sigma_{\rho N}^{2} /(16 \pi B)$ and kept the leading contribution corresponding to the so-called one-step $\rho$ photoproduction process. In the derivation of Eq. (7), we used 
that the nuclear density $\rho_{A}(b, z)$ is a much slower function of the transverse coordinate $b$ than $\Gamma_{N}$, which allowed us to express the answer in a compact form in terms of $T_{A}(b)$. Therefore, the final expression for the $\gamma A \rightarrow \rho A^{\prime}$ quasielastic incoherent cross section reads

$$
\begin{aligned}
\sigma_{\gamma A \rightarrow \rho A^{\prime}}^{\text {Glauber }} & =\left(\frac{e}{f_{\rho}}\right)^{2} \frac{\sigma_{\rho N}^{2}}{16 \pi B} \int d^{2} \vec{b} T_{A}(b) e^{-\sigma_{\rho N}^{\text {in }} T_{A}(b)} \\
& =\sigma_{\gamma p \rightarrow \rho p} \int d^{2} \vec{b} T_{A}(b) e^{-\sigma_{\rho N}^{\text {in }} T_{A}(b)},
\end{aligned}
$$

where $\sigma_{\rho N}^{\text {in }}=\sigma_{\rho N}-\sigma_{\rho N}^{\mathrm{el}}$ is the inelastic $\rho$ meson-nucleon cross section; $\sigma_{\gamma p \rightarrow \rho p}=\left(e / f_{\rho}\right)^{2} \sigma_{\rho N}^{\mathrm{el}}$ is the elastic photoproduction cross section on the nucleon in the VMD model. Equation (8) has a clear physical meaning and interpretation: photoproduction of $\rho$ mesons takes place on any of $A$ nucleons of the target, whose distribution in the transverse plane is given by $T_{A}(b)$, and the produced $\rho$ meson can further interact with the rest of target nucleons. While elastic interactions are allowed, the inelastic re-scattering would destroy the finalstate $\rho$ meson and, hence, should be rejected; the probability not to have inelastic scattering is given by $\exp \left[-\sigma_{\rho N}^{\text {in }} T_{A}(b)\right]$.

Equation (8) was derived assuming independent nucleons in the ground-state nuclear wave function. One can readily go beyond this approximation and take into account the effect of short-range nucleon-nucleon corrections (SRCs) in the nuclear wave function [25-27]. While the SRCs can noticeably modify the $t$ dependence of the incoherent cross section, the $t$-integrated cross section is influenced weakly. Hence, this effect can be safely neglected in our analysis.

Equation (8) implies that nuclear shadowing arises from rescattering of a single state with the cross section $\sigma_{\rho N}$, i.e., that the effect of diffractively produced states leading to the inelastic (Gribov) correction is neglected. A convenient way to take into account the inelastic shadowing correction is offered by the formalism of cross section fluctuations capturing the composite hadronic structure of the photon; see, e.g., [8,9,20]. In this approach [28-31], the key quantity is the distribution $P(\sigma)$ giving the probability for the hadronic component of the photon to interact with the nucleon with the cross section $\sigma$. Following the analysis of Refs. [8,9], we parametrize this distribution in the following form:

$$
P(\sigma)=\frac{C}{1+\left(\sigma / \sigma_{0}\right)^{2}} e^{-\left[\left(\sigma / \sigma_{0}\right)^{2}-1\right]^{2} / \Omega^{2}} .
$$

The free parameters $C, \sigma_{0}$, and $\Omega$ are found from the constraints on the first three moments of the distribution $P(\sigma)$ :

$$
\begin{aligned}
\int_{0}^{\infty} d \sigma P(\sigma) & =1, \\
\int_{0}^{\infty} d \sigma P(\sigma) \sigma & =\hat{\sigma}_{\rho N}\left(W_{\gamma p}\right), \\
\int_{0}^{\infty} d \sigma P(\sigma) \sigma^{2} & =\left[\hat{\sigma}_{\rho N}\left(W_{\gamma p}\right)\right]^{2}\left(1+\omega_{\sigma}\left(W_{\gamma p}\right)\right) .
\end{aligned}
$$

The first equation is the probability conservation. The second equation constrains the average value of $P(\sigma)$ and implies that the hadronic fluctuations of the photon should lead to the effective $\rho$-nucleon cross section $\hat{\sigma}_{\rho N}$. As discussed in Ref. [8], the $\hat{\sigma}_{\rho N}$ effective cross section determined from the fit to the available data on the forward $d \sigma_{\gamma p \rightarrow \rho p}(t=0) / d t$ cross section using the VMD relation,

$$
\hat{\sigma}_{\rho N}\left(W_{\gamma p}\right)=\left(\frac{f_{\rho}^{2}}{4 \pi \alpha_{\mathrm{em}}} 16 \pi d \sigma_{\gamma p \rightarrow \rho p}\left(W_{\gamma p}, t=0\right) / d t\right)^{1 / 2}
$$

turns out to be somewhat smaller than an estimate based on the constituent quark model because of an enhanced contribution of small- $\sigma$ fluctuations. It is reflected in the form of $P(\sigma)$ in Eq. (9) and generally leads to a violation of the naive VMD model. The third equation of Eq. (10) constrains the dispersion of $P(\sigma)$, which is parametrized by $\omega_{\sigma}$. In our analysis, following Ref. [8], we relate it to the corresponding parameter for the pion and use $\omega_{\sigma}=0.3 \pm 0.05$. This uncertainty in $\omega_{\sigma}$ leads to the uncertainty of our predictions of the nuclear cross sections, which we show by red shaded bands in Figs. 4 and 6 below.

In incoherent $\rho$ photoproduction on nuclei, hadronic fluctuations of the photon act at the level of the $\gamma A \rightarrow \rho A^{\prime}$ amplitude. The corresponding quasielastic incoherent cross section can be readily obtained by generalizing the derivation of Eq. (8) [the superscript "GG" stands for Gribov Glauber],

$$
\begin{aligned}
\sigma_{\gamma A \rightarrow \rho A^{\prime}}^{\mathrm{GG}} & =\left(\frac{e}{f_{\rho}}\right)^{2} \int d^{2} \vec{b} \int d \sigma P(\sigma) \int d \sigma^{\prime} P\left(\sigma^{\prime}\right) \frac{\sigma \sigma^{\prime}}{16 \pi B} T_{A}(b) \exp \left[-\frac{\sigma+\sigma^{\prime}}{2} T_{A}(b)+\frac{\sigma \sigma^{\prime}}{16 \pi B} T_{A}(b)\right] \\
& =\left(\frac{e}{f_{\rho}}\right)^{2} \int d^{2} \vec{b} T_{A}(b)\left(\int d \sigma P(\sigma) \frac{\sigma}{\sqrt{16 \pi B}} \exp \left[-\frac{\sigma^{\mathrm{in}}}{2} T_{A}(b)\right]\right)^{2} .
\end{aligned}
$$

To present the answer in a compact form, in the exponential factor in the first line we used that $\sigma \sigma^{\prime} /(16 \pi B)=$ $\left[\sigma^{2} /(16 \pi B)+\sigma^{\prime 2} /(16 \pi B)\right] / 2-\left(\sigma-\sigma^{\prime}\right)^{2} /(32 \pi B)$ and neglected the contribution of the second term, whose contribution is small because both $\sigma$ and $\sigma^{\prime}$ are distributed (fluctuate) around the same average cross section. Neglecting hadronic fluctuations of the photon, i.e., replacing $P(\sigma)$ by the $\delta$ function in Eq. (12), one obtains the Glauber model expression of Eq. (8).
For comparison and completeness, we also give the cross section of coherent $\rho$ photoproduction in the same approach [8],

$$
\sigma_{\gamma A \rightarrow \rho A}^{\mathrm{GG}}=\left(\frac{e}{f_{\rho}}\right)^{2} \int d^{2} \vec{b}\left|\int d \sigma P(\sigma)\left(1-e^{-\frac{\sigma}{2} T_{A}(\vec{b})}\right)\right|^{2} .
$$

In the absence of the fluctuations, i.e., using $P(\sigma)=\delta(\sigma-$ $\left.\sigma_{\rho N}\right)$ in Eq. (13), one obtains the standard expression for the 
coherent $\rho$ photoproduction cross section in the optical limit of the Glauber model.

\section{RESULTS, DISCUSSION, AND COMPARISON TO STARLIGHT}

As follows from the final expressions for the cross section of incoherent $\rho$ photoproduction on heavy nuclei [see Eqs. (8) and (12)], predictions for the nuclear cross section directly depend either on the $t$-integrated $\sigma_{\gamma p \rightarrow \rho p}\left(W_{\gamma p}\right)$ cross section on the proton or on the differential $d \sigma_{\gamma p \rightarrow \rho p}\left(W_{\gamma p}, t=0\right) / d t$ cross section extrapolated to $t=0$; additionally, the effect of nuclear attenuation (shadowing) depends on the slope $B$ of the $t$ dependence of the $\gamma p \rightarrow \rho p$ cross section. In our analysis, we calculate the photoproduction cross section on the proton,

$$
\frac{d \sigma_{\gamma p \rightarrow \rho p}\left(W_{\gamma p}, t\right)}{d t}=\left|T_{\gamma p \rightarrow \rho p}\right|^{2},
$$

using the Donnachie-Landshoff (DL) model [32,33] accounting for contributions of the soft DL Pomeron (P), Reggeon $(\mathrm{R})$, and hard pomeron $(\mathrm{H})$ exchanges in the amplitude $T_{\gamma p \rightarrow \rho p}$,

$$
\begin{aligned}
T_{\gamma p \rightarrow \rho p}(s, t)= & i F_{p}(t) G_{\rho}(t)\left[C_{P} e^{-\frac{1}{2} i \pi \alpha_{P}(t)}\left(2 \alpha_{P}^{\prime} s\right)^{\alpha_{P}(t)-1}\right. \\
& \left.+C_{R} e^{-\frac{1}{2} i \pi \alpha_{R}(t)}\left(2 \alpha_{R}^{\prime} s\right)^{\alpha_{R}(t)-1}\right]+T_{H}(s, t),
\end{aligned}
$$

where $F_{1}^{p}(t)$ and $G_{\rho}(t)$ are the proton Dirac and the $\gamma \rho$ vertex form factors; $\alpha_{P}(t)=\alpha_{P}(0)+\alpha_{P}^{\prime} t$ is the soft Pomeron trajectory; $\alpha_{R}(t)=\alpha_{R}(0)+\alpha_{R}^{\prime} t$ is the Reggeon trajectory; $s=W_{\gamma p}^{2}$. In our calculations, we have used the values of $\alpha_{P}(0)=1.093$, $\alpha_{P}^{\prime}=0.25 \mathrm{GeV}^{-2}$, and $\alpha_{R}(0)=0.5, \alpha_{R}^{\prime}=0.93 \mathrm{GeV}^{-2}$ for the intercepts and the slopes of the soft Pomeron and Reggeon trajectories, respectively. Also, we slightly readjusted the values of the normalization constants $C_{P}$ and $C_{R}$ to describe better the energy dependence of the experimental total and forward cross sections of the $\gamma p \rightarrow \rho p$ process measured in a wide range of energies. The parameters fixing the contribution of the hard Pomeron exchange were taken from [32,33]. Note that the DL hard Pomeron gives a significant contribution to $d \sigma_{\gamma p \rightarrow \rho p}\left(W_{\gamma p}, t\right) / d t$ only for large $-t>1 \mathrm{GeV}^{2}$ and at energies much higher than the considered range. Because our calculation of the $\gamma p \rightarrow \rho p$ photoproduction cross section relies on the vector meson dominance (VMD) and the use of Eq. (15), we refer to this approach as the modified DL (mDL) model.

Figure 1 shows the $\sigma_{\gamma p \rightarrow \rho p}$ cross section as a function of the photon-proton energy $W_{\gamma p}$ : over a broad range of $W_{\gamma p}$, our $\mathrm{mDL}$ model given by the solid curve describes well the available data obtained with fixed targets (SLAC [34], CERN [35], FNAL [36]), at the HERA lepton-proton collider [37-39], and in the proton-nucleus $(p A)$ ultraperipheral collisions (UPCs) by the CMS collaboration at the LHC [40]. Note that the theoretical cross section was linearly extrapolated to the threshold for $W_{\gamma p}<2 \mathrm{GeV}$. It is important to point out that the analysis of the $2020 \mathrm{H} 1$ data gives $\alpha_{P}^{\prime}=0.233_{-0.074}^{+0.067} \mathrm{GeV}^{-2}$ [39], which agrees with the value used in the mDL model (15) employed in our calculations.

The $d \sigma_{\gamma p \rightarrow \rho p}\left(W_{\gamma p}\right) / d t$ differential cross section in the mDL model with $\alpha_{P}^{\prime}=0.15 \mathrm{GeV}^{-2}$ and $\alpha_{P}^{\prime}=0.25 \mathrm{GeV}^{-2}$

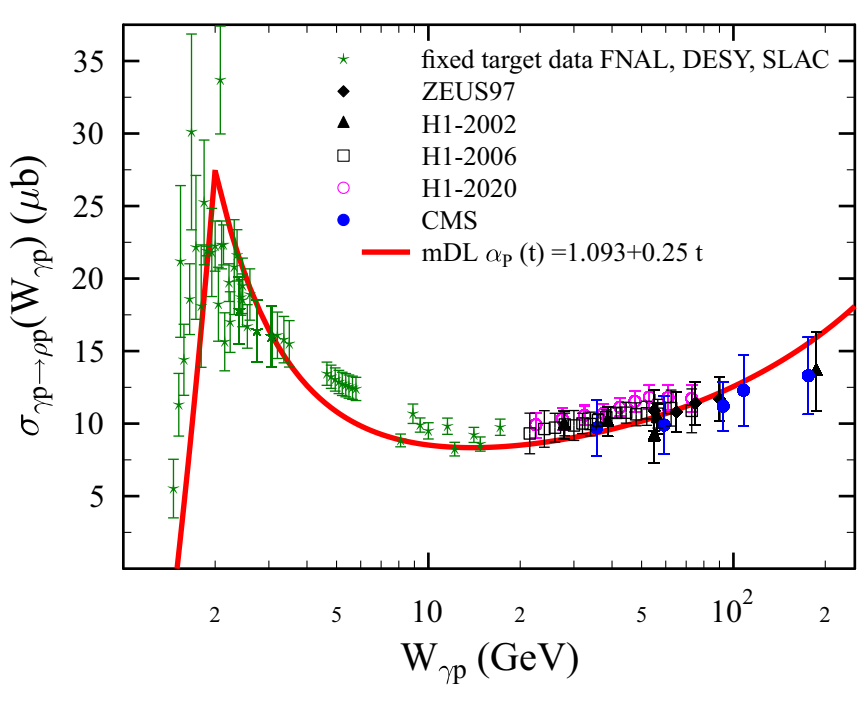

FIG. 1. The $\sigma_{\gamma p \rightarrow \rho p}$ cross section as a function of the photonproton energy $W_{\gamma p}$ : the mDL model vs the available fixed target, HERA, and $p A$ UPC at the LHC data.

values of the slope the Pomeron trajectory is shown in Fig. 2. The results of the model are compared to the $\mathrm{H} 1$ data [38] at $W_{\gamma p}=62.4 \mathrm{GeV}$ (a) and the CMS data [40] at $W_{\gamma p}=59.2$ $\mathrm{GeV}$ (b). These values of $W_{\gamma p}$ correspond to the photonnucleon energy in $\rho$ photoproduction at $y=0$ in $\mathrm{Pb}-\mathrm{Pb}$ UPCs at $\sqrt{s_{N N}}=5.02 \mathrm{TeV}$. One can see from this figure that while the $\mathrm{H} 1$ data favors a steeper $|t|$ dependence with the $\alpha_{P}^{\prime}=0.25 \mathrm{GeV}^{-2}$ slope of the Pomeron trajectory, the recent CMS data [40] seems to prefer the lower value of the slope, $\alpha_{P}^{\prime}=0.15 \mathrm{GeV}^{-2}$. This illustrates the current uncertainty in the value of the $d \sigma_{\gamma p \rightarrow \rho p}\left(W_{\gamma p}, t\right) / d t$ cross section serving as input for the calculation of the cross section of incoherent $\rho$ photoproduction on nuclei.

Our predictions for the cross sections of $\rho$ photoproduction in nucleus-nucleus UPCs in the LHC kinematics are presented in Figs. 3-6.

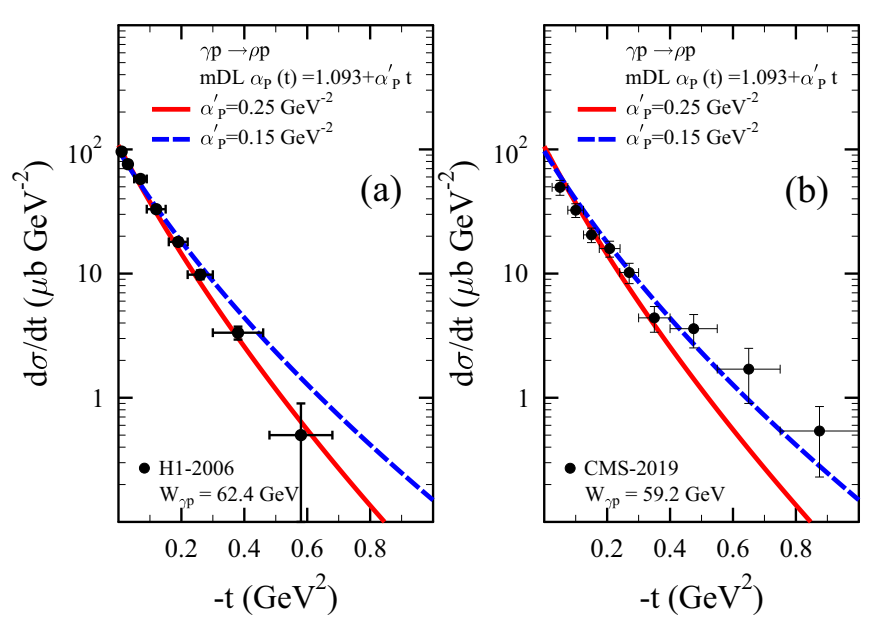

FIG. 2. The $d \sigma_{\gamma p \rightarrow \rho p}\left(W_{\gamma p}\right) / d t$ differential cross section in the mDL model with $\alpha_{P}^{\prime}=0.15 \mathrm{GeV}^{-2}$ and $\alpha_{P}^{\prime}=0.25 \mathrm{GeV}^{-2}$ values of the slope the Pomeron trajectory vs the $\mathrm{H} 1$ data [38] at $W_{\gamma p}=$ 62.4 GeV (a) and the CMS data [40] at $W_{\gamma p}=59.2 \mathrm{GeV}$ (b). 


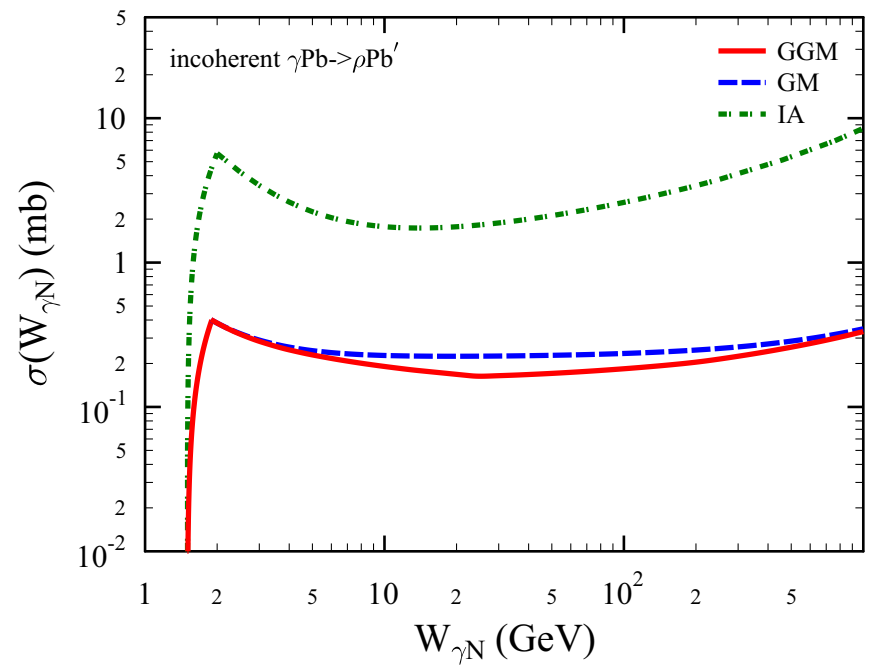

FIG. 3. The $\sigma_{\gamma A \rightarrow \rho A^{\prime}}\left(W_{\gamma p}\right)$ incoherent cross section as a function of $W_{\gamma p}$ in the Gribov-Glauber model (red solid curve labeled "GGM"), the Glauber model (blue dashed curve labeled "GM"), and the impulse approximation (green dot-dashed curve).

Figure 3 shows the $\gamma A \rightarrow \rho A^{\prime}$ incoherent cross section as a function of the photon-nucleon energy $W_{\gamma p}$. The three curves correspond to the results of the calculations using the Gribov-Glauber model of Eq. (12) (the red solid curve labeled "GGM"), the Glauber model of Eq. (8) (the blue dashed curve labeled "GM"), and the impulse approximation (the green dot-dashed curve), where one neglects the effect of nuclear attenuation, $\sigma_{\gamma A \rightarrow \rho A^{\prime}}^{\mathrm{IA}}=A \sigma_{\gamma p \rightarrow \rho p}$. One can see from the figure that the effect of nuclear shadowing is very large and leads to the suppression of the incoherent cross section compared to the impulse approximation by the factor of 10 . Also, one can see that the inelastic nuclear shadowing additionally reduces the incoherent cross section by about $25 \%$ in a broad range of $W_{\gamma p}$. Note that in our calculations in the Gribov-Glauber model, in the interval $20 \leqslant W_{\gamma p} \leqslant 200 \mathrm{GeV}$ we used the nominal central value for the parameter quantifying the photon hadronic fluctuations, $\omega_{\sigma}=0.3$.

For $W_{\gamma p}>200 \mathrm{GeV}$, based on data on inelastic diffraction in antiproton-proton and proton-proton scattering at Tevatron and LHC energies, it is expected that $\omega_{\sigma}$ decreases with an increase of energy and eventually vanishes at asymptotically high energies because of an onset of the so-called black disk limit [8]. This results in a gradual decrease of inelastic nuclear shadowing for very large $W_{\gamma p}$ leading to convergence of the red solid and blue dashed curves. The uncertainty in $\omega_{\sigma}$ leads to a small, of the order of $5 \%$, uncertainty in the predicted incoherent cross section, which is significantly smaller than the difference among the three curves shown in this figure.

Figure 4 shows the incoherent UPC cross section (1) as a function of the $\rho$ meson rapidity $y$ at $\sqrt{s_{N N}}=5.02 \mathrm{TeV}$. The red solid curve corresponds to $\sigma_{\gamma A \rightarrow \rho A^{\prime}}$ in the GribovGlauber model; the blue dashed curve is the result of the Glauber model, c.f. Fig. 3 and its discussion. The shaded band shows the theoretical uncertainty of our predictions due to the uncertainty in the value of $\omega_{\sigma}$, which we take to be $\omega_{\sigma}=0.3 \pm 0.05$ [8]. The black dot-dashed curve is the result

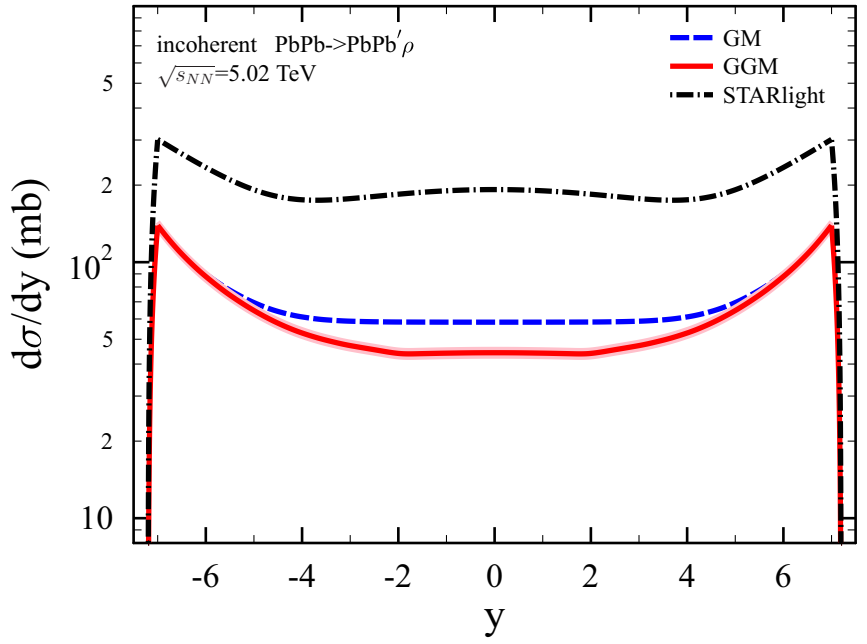

FIG. 4. The incoherent UPC cross section $d \sigma_{A A \rightarrow \rho A A^{\prime}} / d y$ as a function of the $\rho$ meson rapidity $y$ at $\sqrt{s_{N N}}=5.02 \mathrm{TeV}$. Shown are predictions of the Gribov-Glauber (red solid curve with a shaded band), Glauber (blue dashed), and STARlight (black dot-dashed curve) models.

of the STARlight model [14]. One can see that over essentially the entire range of $y$, the predictions of the Gribov-Glauber model lie dramatically lower than those of the STARlight model.

In the STARlight framework, it is assumed that the cross section of incoherent photoproduction of vector mesons on nuclear targets is proportional to the ratio of the inelastic $\rho A$ and $\rho N$ cross sections and is given by the following expression:

$$
\sigma_{\gamma A \rightarrow \rho A^{\prime}}^{\text {STARlight }}=\frac{\sigma_{\gamma p \rightarrow \rho p}}{\sigma_{\rho N}^{\text {in }}} \sigma_{\rho A}^{\text {in }}=\left(\frac{e}{f_{\rho}}\right)^{2} \frac{\sigma_{\rho N}^{\text {el }}}{\sigma_{\rho N}^{\text {in }}} \sigma_{\rho A}^{\text {in }},
$$

where $\sigma_{\rho A}^{\text {in }}$ is calculated in the Glauber model,

$$
\sigma_{\rho A}^{\text {in }}=\int d^{2} \vec{b}\left(1-e^{-\sigma_{\rho N} T_{A}(b)}\right) .
$$

Equation (16) does not correspond to the Glauber expression for the quasielastic incoherent $\gamma A \rightarrow \rho A^{\prime}$ cross section. As follows from unitary of the Glauber theory, the inelastic $\rho A$ cross section can be presented by a sum of partial cross sections of the inelastic interactions of the produced $\rho$ meson with $N_{\text {eff }}$ nucleons $\left(1 \leqslant N_{\text {eff }} \leqslant A\right)$ [41], which lead to the final state with a much higher multiplicity than that in the incoherent cross section studied in UPCs. As a result, the STARlight predictions for the cross section of incoherent photoproduction of $\rho$ mesons in Pb-Pb UPCs at $\sqrt{s_{N N}}=$ $5.02 \mathrm{TeV}$ significantly overestimate those obtained in our GGM approach (see also Table I).

It is also illustrated by Fig. 5, which shows the incoherent $d \sigma_{A A \rightarrow \rho A A^{\prime}} / d y$ UPC cross sections as a function of the centerof-mass energy $W_{N N}=\sqrt{s_{N N}}$ at the central rapidity $y=0$ and compares the results of the Gribov-Glauber and STARlight models. One can see from this figure that the STARlight predictions are several-fold larger than those of the GribovGlauber model. 
TABLE I. Predictions for the incoherent $d \sigma_{A A \rightarrow \rho A A^{\prime}} / d y$ and coherent $d \sigma_{A A \rightarrow \rho A A} / d y$ cross sections (in $\mathrm{mb}$ ) of $\rho$ photoproduction in $\mathrm{Pb}-\mathrm{Pb}$ UPCs at $\sqrt{s_{N N}}=5.02 \mathrm{TeV}$ and $y=0$ in the framework presented in this paper (GM and GGM) and the STARlight model.

\begin{tabular}{lccc}
\hline \hline & GM & GGM & STARlight \\
\hline Incoherent, mb & 58 & 44 & 192 \\
Coherent, mb & 840 & 570 & 440 \\
\hline \hline
\end{tabular}

The quasielastic incoherent cross sections of Eqs. (8) and (12) do not include the contribution of $\rho$ photoproduction with nucleon dissociation, $\gamma N \rightarrow \rho Y$, where $Y$ denotes the hadronic system with mass $M_{Y}$. If this contribution is not rejected experimentally, it will increase the incoherent cross section. The effect can be taken into account using the approach developed for incoherent $J / \psi$ photoproduction on nuclei [42]. Using this method, the cross section of incoherent $\rho$ photoproduction on nuclei, which includes both elastic and nucleon-dissociative photoproduction on target nucleons, can be presented in the following form [compare to Eq. (12)]:

$$
\begin{aligned}
\sigma_{\gamma A \rightarrow \rho A^{\prime}+Y}^{\mathrm{GG}}= & \left(1+\frac{\sigma_{\gamma p \rightarrow \rho Y}}{\sigma_{\gamma p \rightarrow \rho p}}\right)\left(\frac{e}{f_{\rho}}\right)^{2} \int d^{2} \vec{b} T_{A}(b) \\
& \times\left(\int d \sigma P(\sigma) \frac{\sigma}{\sqrt{16 \pi B}} \exp \left[-\frac{\sigma^{\text {in }}}{2} T_{A}(b)\right]\right)^{2},
\end{aligned}
$$

where $\sigma_{\gamma p \rightarrow \rho p}$ and $\sigma_{\gamma p \rightarrow \rho Y}$ are the $t$-integrated cross sections of elastic and nucleon-dissociative $\rho$ photoproduction on the proton, respectively. Using the ZEUS analysis of elastic and proton-dissociative $\rho^{0}$ photoproduction at HERA [37]

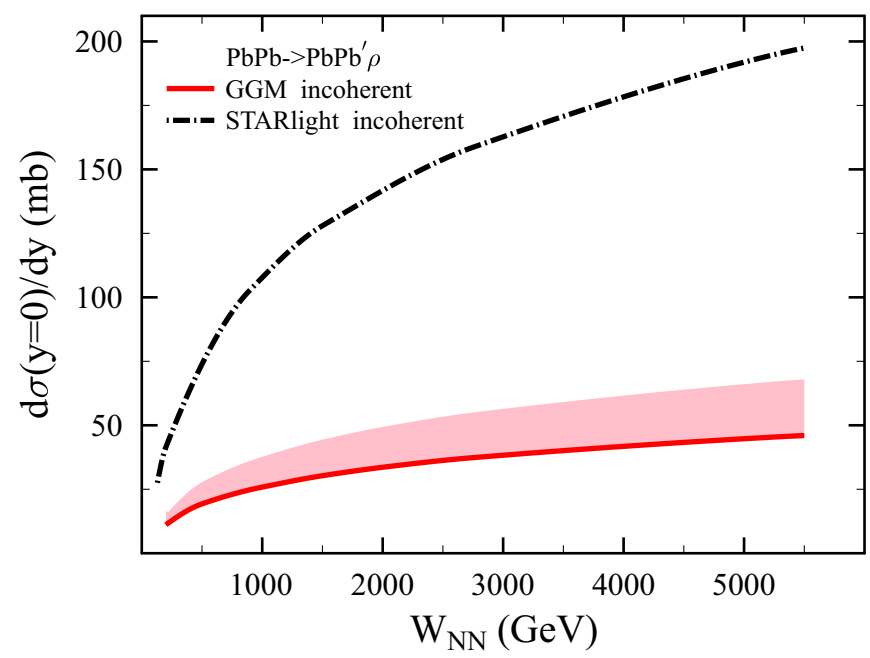

FIG. 5. The incoherent UPC cross sections as a function of $W_{N N}=\sqrt{s_{N N}}$ at $y=0$ in the Gribov-Glauber (red solid) and STARlight (black dot-dashed) models. The red shaded band shows the range of predictions for the cross section of incoherent $\rho$ photoproduction on nuclei, which includes both elastic and nucleondissociative photoproduction on target nucleons [see Eq. (18)]. that found $\sigma_{\gamma p \rightarrow \rho p} / \sigma_{\gamma p \rightarrow \rho Y}=2.0 \pm 0.2$ (stat.) \pm 0.7 (syst.) in kinematic domain $M_{Y}<0.1 W_{\gamma p}^{2}$ and $|t|<0.5 \mathrm{GeV}^{2}$, we estimate that the nucleon dissociation may increase the cross section of one-step incoherent $\rho$ photoproduction by as much as $50 \%$. The exact magnitude of this contribution depends on such data selection criteria as the mass of the produced state $Y$ and the range of the momentum transfer $t$. To reflect it, the possible range of our predictions for $\sigma_{\gamma A \rightarrow \rho A^{\prime}+Y}^{\mathrm{GG}}$ of Eq. (18) is given by the red shaded band in Fig. 5. Note that the uncertainty in the value of $\omega_{\sigma}$ results in a small, 5\% uncertainty in the predicted incoherent cross section, which can be neglected compared to the magnitude of the nucleondissociation contribution.

Note that predictions of the Glauber model fall within the range of the shaded band and, hence, are not shown; the difference between the Gribov-Glauber and Glauber model predictions can be readily read off Fig. 4 .

Predictions for the cross section of incoherent $\rho$ photoproduction in $\mathrm{Pb}-\mathrm{Pb}$ UPCs in the LHC kinematics including the effect of nucleon dissociation were also made in the hot-spot model in Ref. [15]. While the relative magnitude of the proton-dissociative contribution to the incoherent cross section is similar to our result, the absolute value of the incoherent cross section is several times smaller than our estimate. Thus, future measurements of incoherent $\rho$ photoproduction in heavy-ion UPCs at the LHC will help to discriminate between the discussed approaches and constrain the dynamics of nuclear shadowing in vector meson photoproduction on nuclei.

It is also instructive to compare the incoherent and coherent cases. The cross section of coherent $\rho$ photoproduction on nuclei in the STARlight model is given by the following expression:

$$
\begin{aligned}
\sigma_{\gamma A \rightarrow \rho A}^{\text {STARlight }} & =\frac{d \sigma_{\gamma A \rightarrow \rho A}(t=0)}{d t} \int_{\left|t_{\min }\right|}^{\infty} d t F_{A}^{2}(t) \\
& =\left(\frac{e}{f_{\rho}}\right)^{2} \frac{\sigma_{\rho A}^{2}}{16 \pi} \int_{\left|t_{\min }\right|}^{\infty} d t F_{A}^{2}(t),
\end{aligned}
$$

where $\left|t_{\min }\right|=\left(M_{\rho}^{2} m_{N} / W_{\gamma p}\right)^{2}$. The factorized form of the expression in Eq. (19) [compare to Eq. (13) in the GribovGlauber model] assumes that the $t$ dependence of the amplitude of coherent photoproduction on nuclei can be approximated by the undistorted nuclear form factor $F_{A}(t)$. This assumption disagrees with the Glauber model (see, e.g., [6]) and does not only result in the $t$ dependence of the $d \sigma_{A A \rightarrow \rho A A} /(d y d t)$ cross section, which is wider than that predicted in the Gribov-Glauber approach [8] and seemed to be observed in the data [17], but also increases the $t$ integrated cross section by a factor about 1.3-1.4. At the same time, in the standard option of the STARlight model, the $\sigma_{\rho A}$ cross section in Eq. (19) is identified with the inelastic $\sigma_{\rho A}^{\text {in }}$ cross section (17) instead of the total one. It leads to a strong energy-dependent violation of the optical theorem and a suppression of the coherent cross section by approximately a factor of three in the LHC kinematics (a factor of four in the asymptotic black body limit). An interplay of these two effects results in an overall suppression by approximately a 


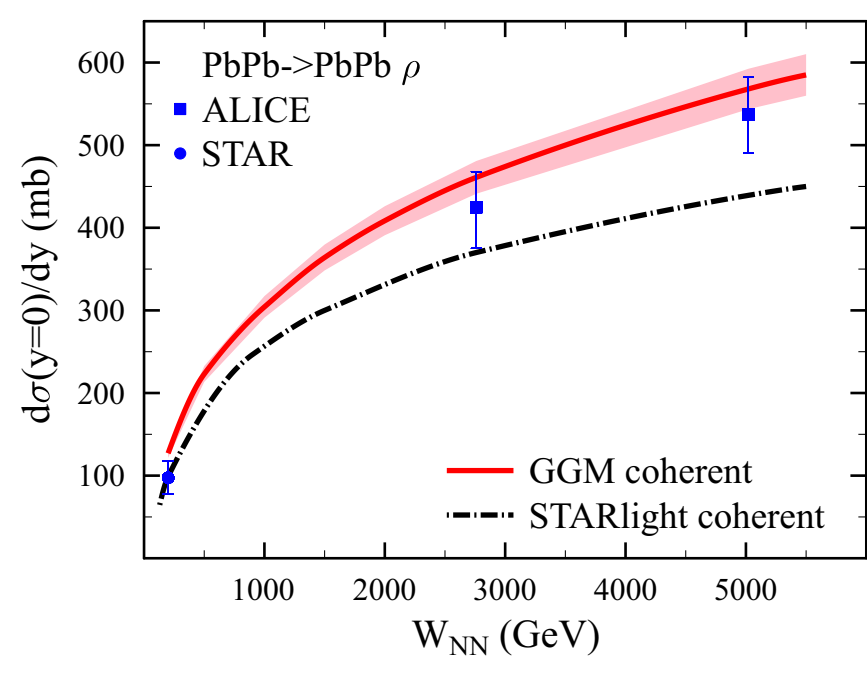

FIG. 6. The coherent UPC cross sections as a function of $W_{N N}=$ $\sqrt{s_{N N}}$ at $y=0$ in the Gribov-Glauber (red solid curve with a shaded band) and STARlight (black dot-dashed curve) models. The scaled STAR measurement at $\sqrt{s_{N N}}=200 \mathrm{GeV}$ [43] and the ALICE measurements at $\sqrt{s_{N N}}=2.76 \mathrm{TeV}$ [17] and $\sqrt{s_{N N}}=5.02 \mathrm{TeV}$ [18] are shown by the filled circle and the squares with error bars, respectively.

factor of two of the cross section of coherent $\rho$ photoproduction on nuclei in the STARlight model compared to the standard optical-limit Glauber model (see Table I). Note that the STARlight framework has an option for the calculation of the $\sigma_{\gamma A \rightarrow \rho A}^{\text {STARlight }}$ cross section with the total $\rho$-nucleus cross section calculated in the Glauber model. It leads to a very large value of the coherent cross section at $\sqrt{s_{N N}}=5.02 \mathrm{TeV}$, $d \sigma_{A A \rightarrow \rho A A}^{\text {STARlight }} / d y(y=0) \approx 1100 \mathrm{mb}$.

Predictions of the Gribov-Glauber and STARlight models for the coherent $d \sigma_{A A \rightarrow \rho A A} / d y$ UPC cross section as a function of $W_{N N}=\sqrt{s_{N N}}$ at $y=0$ are shown in Fig. 6. Also, the scaled results of the STAR measurement of coherent $\rho$ photoproduction in Au-Au UPCs at $\sqrt{s_{N N}}=200 \mathrm{GeV}$ [43] and ALICE measurements of coherent $\rho$ photoproduction in Pb-Pb UPCs at $\sqrt{s_{N N}}=2.76 \mathrm{TeV}$ [17] and $\sqrt{s_{N N}}=5.02 \mathrm{TeV}$ [18] are shown by the filled circle and the squares with error bars, respectively. One can see that the predictions of our approach are in excellent agreement with the ALICE data. Note that the STAR data point for $\mathrm{Au}$ was scaled to $\mathrm{Pb}$ by the ratio of the theoretical cross sections. The Glauber model prediction (not shown) significantly exceeds that of the Gribov-Glauber approach and, hence, fails to describe the Run 1 and 2 ALICE data points (see Ref. [8] and Table I of the present work).

Table I summarizes the results for the incoherent $d \sigma_{A A \rightarrow \rho A A^{\prime}} / d y$ and coherent $d \sigma_{A A \rightarrow \rho A A} / d y$ cross sections of $\rho$ photoproduction in $\mathrm{Pb}-\mathrm{Pb}$ UPCs at $\sqrt{s_{N N}}=5.02 \mathrm{TeV}$ and $y=0$ in the framework presented in this paper (GM and GGM) and the STARlight model. It clearly demonstrates large differences between predictions of the Gribov-Glauber model superseding the Glauber model and those of STARlight, which are especially dramatic for the incoherent cross section.

\section{CONCLUSIONS}

In this paper, using the Gribov-Glauber model for photonnucleus scattering and a generalization of the VMD model for the hadronic structure of the photon, we consider incoherent photoproduction of $\rho$ mesons on heavy nuclei and make predictions for the incoherent $P b P b \rightarrow \rho P b A^{\prime}$ UPC cross section in the LHC kinematics. We present our results as a function of the rapidity $y$ at $\sqrt{s_{N N}}=5.02 \mathrm{TeV}$ and the invariant collision energy $\sqrt{s_{N N}}$ at $y=0$. We also give predictions for the incoherent photoproduction cross section $\gamma P b \rightarrow \rho A^{\prime}$ as a function of the invariant photon-nucleon energy $W_{\gamma p}$. We demonstrate that the effect of the inelastic nuclear shadowing in the incoherent cross sections is significant and leads to an additional 25\% suppression of the cross section. Comparing our predictions to those of the STARlight Monte Carlo framework, we find very significant differences.
[1] A. J. Baltz et al., Phys. Rep. 458, 1 (2008).

[2] R. J. Glauber, Phys. Rev. 100, 242 (1955).

[3] R. J. Glauber and G. Matthiae, Nucl. Phys. B 21, 135 (1970).

[4] V. N. Gribov, Zh. Eksp. Teor. Fiz. 56, 892 (1969) [Sov. Phys. JETP 29, 483 (1969)].

[5] L. Frankfurt, M. Strikman, and M. Zhalov, Phys. Lett. B 537, 51 (2002).

[6] L. Frankfurt, M. Strikman, and M. Zhalov, Phys. Rev. C 67, 034901 (2003).

[7] V. Rebyakova, M. Strikman, and M. Zhalov, Phys. Lett. B 710, 647 (2012).

[8] L. Frankfurt, V. Guzey, M. Strikman, and M. Zhalov, Phys. Lett. B 752, 51 (2016).

[9] V. Guzey, E. Kryshen, and M. Zhalov, Phys. Rev. C 93, 055206 (2016).

[10] V. P. Gonçalves and M. V. T. Machado, Phys. Rev. C 84, 011902(R) (2011).
[11] G. Sampaio dos Santos and M. V. T. Machado, Phys. Rev. C 91, 025203 (2015).

[12] V. P. Gonçalves, M. V. T. Machado, B. D. Moreira, F. S. Navarra and G. S. dos Santos, Phys. Rev. D 96, 094027 (2017).

[13] S. R. Klein and J. Nystrand, Phys. Rev. C 60, 014903 (1999).

[14] S. R. Klein, J. Nystrand, J. Seger, Y. Gorbunov, and J. Butterworth, Comput. Phys. Commun. 212, 258 (2017).

[15] J. Cepila, J. G. Contreras, M. Krelina and J. D. Tapia Takaki, Nucl. Phys. B 934, 330 (2018).

[16] V. A. Khoze, A. D. Martin, and M. G. Ryskin, J. Phys. G 46, 085002 (2019).

[17] J. Adam et al. (ALICE Collaboration), J. High Energy Phys. 09 (2015) 095.

[18] S. Acharya et al. (ALICE Collaboration), J. High Energy Phys. 06 (2020) 035.

[19] V. M. Budnev, I. F. Ginzburg, G. V. Meledin, and V. G. Serbo, Phys. Rep. 15, 181 (1975). 
[20] V. Guzey, M. Strikman, and M. Zhalov, Eur. Phys. J. C 74, 2942 (2014).

[21] M. Vidovic, M. Greiner, C. Best, and G. Soff, Phys. Rev. C 47, 2308 (1993).

[22] M. Beiner, H. Flocard, N. van Giai, and P. Quentin, Nucl. Phys. A 238, 29 (1975).

[23] M. Tanabashi et al. (Particle Data Group), Phys. Rev. D 98 , 030001 (2018).

[24] T. H. Bauer, R. D. Spital, D. R. Yennie, and F. M. Pipkin, Rev. Mod. Phys. 50, 261 (1978); 51, 407(E) (1979).

[25] E. J. Moniz and G. D. Nixon, Ann. Phys. 67, 58 (1971).

[26] G. Von Bochmann, B. Margolis, and C. L. Tang, Phys. Lett. B 30, 254 (1969).

[27] D. R. Yennie, Cargese Lect. Phys. 7, 331 (1977).

[28] M. L. Good and W. D. Walker, Phys. Rev. 120, 1857 (1960).

[29] H. I. Miettinen and J. Pumplin, Phys. Rev. D 18, 1696 (1978).

[30] B. Z. Kopeliovich, L. I. Lapidus, and A. B. Zamolodchikov, Pisma Zh. Eksp. Teor. Fiz. 33, 612 (1981) [JETP Lett. 33, 595 (1981)].
[31] B. Bläettel, G. Baym, L. L. Frankfurt, H. Heiselberg, and M. Strikman, Phys. Rev. D 47, 2761 (1993).

[32] A. Donnachie and P. V. Landshoff, Phys. Lett. B 478, 146 (2000).

[33] A. Donnachie and P. V. Landshoff, arXiv:0803.0686.

[34] J. Park et al., Nucl. Phys. B 36, 404 (1972).

[35] D. Aston et al., Nucl. Phys. B 209, 56 (1982).

[36] R. M. Egloff et al., Phys. Rev. Lett. 43, 657 (1979).

[37] J. Breitweg et al. (ZEUS Collaboration), Eur. Phys. J. C 2, 247 (1998).

[38] R. M. Weber, Report No. DISS-ETH-16709 (ETH Zurich, Zurich, 2006).

[39] V. Andreev et al. (H1 Collaboration), arXiv:2005.14471.

[40] A. M. Sirunyan et al. (CMS Collaboration), Eur. Phys. J. C 79, 702 (2019).

[41] L. Bertocchi and D. Treleani, J. Phys. G 3, 147 (1977).

[42] V. Guzey, M. Strikman, and M. Zhalov, Phys. Rev. C 99, 015201 (2019).

[43] B. I. Abelev et al. (STAR Collaboration), Phys. Rev. C 77, 034910 (2008). 